\title{
The ex utero intrapartum treatment (EXIT) procedure: maternal and fetal considerations
}

Vinod Chinnappa MBBS MD FCARCSI, Stephen H. Halpern MD MSc FRCPC

$\mathrm{R}$ ECENT advances in imaging technology have greatly improved our ability to diagnose anatomic fetal anomalies. Abnormalities immediately after delivery. Some of these abnormalities are amenable to intrapartum surgical treatment. The ex utero intrapartum treatment (EXIT), which has also been described as "operation on placental support" or "airway management on placental support", relieves or bypasses the obstruction while the fetus is still being oxygenated by the utero-placental circulation. Traditionally, this procedure is performed while the mother is deeply anesthetized with a halogenated agent. In this issue of the Journal, George et al. ${ }^{1}$ report the use of regional anesthesia. In this editorial we will examine the EXIT procedure and discuss the requirements for a successful outcome for both the mother and the newborn.

The first EXIT procedure was performed by Norris et al. ${ }^{2}$ in 1989 for preterm delivery of a fetus with a large anterior neck mass. Since that time, the indications for the procedure have expanded to include other extrinsic obstructions, congenital high airway obstruction syndrome and hypoplastic craniofacial syndromes. The removal of tracheal clips placed in utero for the treatment of congenital diaphragmatic hernia is also amenable to the EXIT procedure. Rarely, the procedure has been used before placing the neonate on an external membrane oxygenator, redelivery of chest masses, or as part of the strategy to separate conjoined twins. ${ }^{3}$

The EXIT procedure is very different from routine Cesarean delivery. During a Cesarean delivery it is important to minimize placental transfer of depressant anesthetic agents and to maximize uterine tone immediately after delivery. In contrast, during the EXIT procedure, fetal anesthesia may be desirable and uterine relaxation is essential in order to prevent premature placental separation and the interruption of oxygenation. General anesthesia with high concentrations of a halogenated agent can accomplish both of these goals. ${ }^{4}$ Desflurane is attractive for this purpose because maternal blood levels can be adjusted rapidly and uterine tone can be restored quickly after delivery. ${ }^{5}$ This advantage is particularly noticeable when the EXIT procedure is expected to take a significant length of time. In addition, inhalational agents cross the placenta to provide fetal anesthesia if required. Regional anesthesia, as described by George et al. ${ }^{1}$ with a continuous nitroglycerin infusion and supplemental im fetal anesthesia may also be effective for short procedures.

During the procedure, uterine blood flow must be maintained and optimized. Since maternal blood pressure is a primary determinant of uterine blood flow, normal blood pressure should be meticulously preserved. An arterial line may be useful for this purpose. Maternal hypotension may occur for a number of reasons. Some of these can be corrected by appropriate positioning to avoid aortocaval compression and the use of fluids to ensure adequate intravascular volume. Vasodilation due to inhalational agents or other uterine relaxants can be treated with a vasopressor. Phenylephrine may be a better choice than ephedrine in this situation because it may reduce the incidence of fetal acidosis. ${ }^{6}$ Fetal oxygenation may be further optimized by delivering at least $50 \%$ oxygen to the mother. ${ }^{7}$ Uterine distension should be maintained to avoid premature placental separation. This can be accomplished by delivering only the head of the fetus, leaving the body and umbilical cord in utero. This will also help maintain the body temperature of the fetus during long procedures. In some cases, fluid may be infused into the uterus to maintain intrauterine volume.

CAN J ANESTH $2007 / 54: 3 /$ pp 171-175

From the Department of Anesthesia, Sunnybrook and Women's College Hospitals, University of Toronto, Toronto, Ontario, Canada. Address correspondence to: Dr. Stephen Halpern, Department of Anesthesia, Women's College Hospital 76 Grenville St. Toronto, Ontario M5S 1B2, Canada. Phone: 416-323-6269; Fax: 416-323-2666; E-mail: stephen.halpern@sunnybrook.ca 
Uterine relaxation is an important component of the anesthetic management. Many agents have been used; each has its advantages and disadvantages. Potent inhalational agents and nitroglycerin are used in centres with the most experience with this procedure. Some institutions add preoperative indomethacin. ${ }^{8}$ The use of beta-sympathomimetics and magnesium sulfate have also been described.

In many cases, the EXIT procedure can be considered to be a form of fetal surgery and fetal anesthesia is necessary when invasive procedures are planned. It is well established that the central nervous system of the near-term fetus is capable of transmitting pain impulses and that conscious perception of pain, represented by tests of cortical function, may be present. ${ }^{9}$ One of the cases presented by George et al. ${ }^{1}$ received im ketamine. Other investigators rely on the transplacental passage of inhalational agents. Nondepolarizing muscle relaxants can be directly injected into the fetus if immobilization is a requirement.

Fetal/newborn monitoring may be technically difficult. Some investigators report the use of a pulse oximeter and attempt to maintain fetal oxygen saturation over $40 \%{ }^{3}$ Continuous fetal echocardiography has also been described to detect fetal bradycardia, decreased myocardial contractility, ductal closure and valvular competence. ${ }^{3}$ Finally, it is important to obtain fetal $i v$ access if the procedure is prolonged.

Severe maternal complications may occur. For example, the uterine incision must avoid the placenta. Therefore the uterine scar may be located in an unusual location, resulting in bleeding or uterine rupture during a subsequent pregnancy. In a series of 31 patients, the average estimated blood loss was about $850 \mathrm{~mL} .{ }^{10}$ There were only two maternal complications reported in this series - one patient had a severe hemorrhage and a second patient suffered a uterine dehiscence with a subsequent pregnancy. Other investigators compared the incidence of maternal complications in 34 patients who had the EXIT procedure compared to 32 matched controls who had a Cesarean delivery without an EXIT procedure. ${ }^{11}$ In patients who had the EXIT procedure, the operative time was longer (mean $110 \mathrm{~min} v 557 \mathrm{~min} P<0.0001$ ) and the estimated blood loss was higher (mean $1104 \mathrm{~mL}$ vs $883 \mathrm{~mL} P<0.001)$. Three women in the EXIT group received a blood transfusion compared to one in the Cesarean delivery group. The incidence of wound complications was also higher in the EXIT group ( $15 \%$ vs $2 \%, P=0.03$ ). Of note, the time to discharge was the same in both groups. Neither of these studies is sufficiently large to reliably estimate the incidence short-term or long-term maternal morbidity.
The eventual fetal outcome depends on the primary diagnosis, the extent of the airway anomalies and the severity of associated anomalies. In the series cited above, only one neonate died during the EXIT procedure. ${ }^{10}$ However, to obtain optimal results, it is necessary to assemble an experienced team composed of pediatric surgeons, obstetricians, anesthesiologists, neonatologists, geneticists and ultrasonographers. The operating room becomes crowded with two complete surgical teams and two sets of surgical instruments. Appropriate equipment needs to be readily available. This includes a specially designed uterine stapler with absorbable staples ${ }^{12}$ to avoid hemorrhage during the time of prolonged uterine atony.

Each member of the team has a vital role to play. The obstetrician must avoid damage to the placenta and plans the uterine incision after a careful ultrasonic mapping of the position of the placental edges. After hysterotomy, exposure of the fetus allows for additional im fetal anesthetic if necessary. Once the fetal head is exposed, the airway is secured by rigid bronchoscopy after exposing the larynx. Depending on the indication for the EXIT procedure, the fetus can be exposed as needed. Ultrasound can be used intraoperatively to monitor umbilical cord pulsations and fetal cardiac function. After the procedure, the umbilical cord is cut and the newborn is given to the neonatologist for further evaluation and treatment.

In addition, ethical issues may arise. Maternal risk must be weighed against the potential advantage to the neonate, particularly in cases where other, severe anomalies or genetic defects are present. Each patient requires a case conference involving all members of the team described above. A morphologic and genetic diagnosis is important to ensure that the procedure is not performed on a fetus that has additional lethal anomalies.

The EXIT procedure is relatively new and holds promise for newborns with airway obstruction. The anesthetic can be conducted in a number of ways provided fetal oxygenation and uterine relaxation are maintained. Preoperative planning and communication are the most important factors for success to determine the resources required. The ethical issues must be considered in order to optimize benefit to both the mother and fetus. 


\section{La procédure EXIT (ex utero intrapartum treatment) : considéra- tions foetales et maternelles}

Les progrès récents des technologies d'imagerie ont amélioré de façon significative notre capacité à diagnostiquer les anomalies anatomiques fotales. En général, les anomalies causant une obstruction mécanique des voies respiratoires supérieures ou inférieures sont fatales immédiatement après l'accouchement. Certaines de ces anomalies peuvent être traitées chirurgicalement en intrapartum. Le traitement EXIT, également décrit comme "opération avec assistance placentaire » ou «assistance respiratoire avec soutien placentaire ", soulage ou évite l'obstruction pendant que le fotus est encore oxygéné par la circulation utéro-placentaire. Traditionnellement, cette procédure est effectuée lorsque la mère est profondément anesthésiée à l'aide d'un agent halogéné. Dans ce numéro du Journal, George et coll. ${ }^{1}$ présentent des cas où une anesthésie régionale a été utilisée. Dans cet éditorial, nous allons examiner la procédure EXIT ainsi que les conditions requises pour qu'elle soit bénéfique pour la mère et le nouveau-né.

La première procédure EXIT a été effectuée par Norris et coll. ${ }^{2}$ en 1989 , dans le cas de la naissance avant terme d'un foetus présentant une grosse masse à la partie antérieure du cou. Depuis, cette procédure a également été utilisée dans d'autres cas d'obstructions extrinsèques, de syndrome congénital d'obstruction des voies respiratoires supérieures et de syndromes cranio-faciaux hypoplasiques. L'extraction des pinces trachéales placées in utero pour le traitement d'une hernie diaphragmatique congénitale est aussi effectuée avec la procédure EXIT. La procédure a également été utilisée, quoique rarement, avant de placer le nouveauné sous un oxygénateur externe, lors d'extractions de masses pulmonaires, ou dans le cadre d'une stratégie de séparation de jumeaux conjoints. ${ }^{3}$

La procédure EXIT est très différente d'un accouchement de routine par césarienne. Lors d'un accouchement par césarienne, il est important de minimiser le transfert placentaire d'agents anesthésiques dépresseurs et de maximiser le tonus utérin immédiatement après l'accouchement. En revanche, lors d'un accouchement par procédure EXIT, l'anesthésie fotale peut être souhaitable et la relaxation utérine est indispensable pour éviter une séparation placentaire précoce et l'interruption de l'oxygénation. Ces deux objectifs peuvent être atteints avec une anesthésie générale à fortes concentrations d'agent halogéné. ${ }^{4}$ Le desflurane est intéressant parce que sa concentration plasmatique maternelle peut être ajustée rapidement permettant le rétablissement rapide du tonus utérin après l'accouchement. ${ }^{5}$ Cet aspect est particulièrement avantageux lorsque la procédure EXIT est longue. De plus, les agents par inhalation traversent le placenta afin de fournir, si nécessaire, une anesthésie pour le foetus. Une anesthésie régionale telle que celle décrite par George et coll. ${ }^{1}$, comprenant une perfusion continue de nitroglycérine et une anesthésie foetale supplémentaire par voie intramusculaire, peut aussi être efficace lors d'interventions courtes.

Pendant la procédure, le débit sanguin utérin doit être maintenu et optimisé. Étant donné que la tension artérielle de la mère est un facteur déterminant du débit sanguin de l'utérus, une tension artérielle normale doit être soigneusement préservée. Dans ce but, un cathéter artériel s'avère parfois utile. L'hypotension maternelle peut survenir pour une multitude de raisons. Certaines peuvent être corrigées par un positionnement adéquat évitant la compression aorto-cave et par l'utilisation de liquides afin d'assurer un volume intra-vasculaire adéquat. La vasodilatation causée par les agents par inhalation ou par d'autres relaxants utérins peut être traitée à l'aide d'un vasopresseur. Dans cette situation, la phényléphrine est peut-être à préférer à l'éphédrine, parce qu'elle réduit l'incidence d'acidose fortale. ${ }^{6}$ L'oxygénation fotale peut être optimisée en donnant au moins $50 \%$ d'oxygène à la mère. ${ }^{7}$ La distension utérine devrait être maintenue afin d'éviter une séparation placentaire précoce. Pour ce faire, on peut sortir uniquement la tête du fotus en laissant le corps et le cordon ombilical in utero. Ceci permettra également de maintenir la température corporelle du foetus lors de longues interventions. Dans certains cas, du liquide peut être perfusé dans l'utérus afin de maintenir le volume intra-utérin.

La relaxation de l'utérus est une composante importante de la prise en charge anesthésique. De nombreux agents ont été utilisés, et chacun présente des avantages et des inconvénients. De puissants agents par inhalation et de la nitroglycérine sont utilisés dans les centres les plus expérimentés avec cette procédure. Dans certaines institutions, on ajoute de l'indométhacine en préopératoire. ${ }^{8}$ L'utilisation de bêta-sympathicomimétiques et de sulfate de magnésium a également été décrite. 
Dans de nombreux cas, la procédure EXIT peut être considérée comme une forme de chirurgie foetale, et une anesthésie foetale est nécessaire lorsque des procédures invasives sont prévues. Il est clairement établi que le système nerveux central du foetus proche du terme est capable de transmettre des impulsions de douleur et que la perception consciente de la douleur est possible, comme le témoignent des examens de la fonction corticale. ${ }^{9}$ Une des patientes de l'étude de George et coll. ${ }^{1}$ a reçu de la kétamine en $\mathrm{im}$. D'autres chercheurs se sont appuyés sur le passage trans-placentaire d'agents par inhalation. Des curares nondépolarisants peuvent être injectés directement dans le fotus si l'immobilisation est nécessaire.

Le monitorage du foetus ou du nouveau-né peut être techniquement difficile. Certains chercheurs font état de l'utilisation d'un saturomètre et tentent de maintenir la saturation d'oxygène foetale au-dessus de $40 \%{ }^{3}$ L'échocardiographie foetale continue a également été utilisée pour détecter une bradycardie foetale, une contractilité du myocarde réduite, la fermeture du canal artériel et la compétence valvulaire. ${ }^{3}$ Enfin, il est important d'obtenir un accès $i v$ foetal si l'intervention se prolonge.

Des complications maternelles graves peuvent se produire. Par exemple, l'incision utérine doit éviter le placenta. C'est pourquoi la cicatrice utérine se situe parfois dans un endroit inhabituel, ce qui peut avoir pour conséquence des saignements ou une rupture utérine lors d'une grossesse ultérieure. Dans une série de 31 patientes, la perte de sang moyenne estimée a été d'environ $850 \mathrm{~mL} .{ }^{10} \mathrm{Il}$ n'y a eu que de deux complications maternelles dans cette série - une patiente a eu une hémorragie grave et, lors d'une grossesse suivante, la seconde a eu une déhiscence utérine. D'autres chercheurs ont comparé l'incidence des complications maternelles chez 34 patientes ayant eu la procédure EXIT en comparaison à 32 témoins appariés qui ont eu un accouchement par césarienne sans procédure EXIT. ${ }^{11}$ Chez les patientes en procédure EXIT, l'opération était plus longue (moyenne $110 \mathrm{~min}$ vs $57 \mathrm{~min} P<0,0001$ ) et la perte de sang estimée plus élevée (moyenne $1104 \mathrm{~mL}$ vs $883 \mathrm{~mL}$ $P<0,001)$. Trois femmes du groupe EXIT ont reçu une transfusion sanguine comparativement à une dans le groupe d'accouchement par césarienne. L'incidence de complications de plaie était également plus élevée dans le groupe EXIT $(15 \%$ ps $2 \%, P=0,03)$. Il est à noter que le temps jusqu'au congé était le même dans les deux groupes. Aucune de ces études n'est suffisamment étendue pour estimer de façon fiable l'incidence de morbidité maternelle à court et à long terme.

L'évolution finale du fotus dépend du diagnostic primaire, de l'étendue des anomalies des voies respiratoires et de la gravité des anomalies liées. Dans la série citée ci-dessus, un nouveau-né seulement est mort durant la procédure EXIT. ${ }^{10}$ Toutefois, afin d'obtenir des résultats optimaux, il est nécessaire de réunir une équipe expérimentée composée de chirurgiens pédiatriques, d'obstétriciens, d'anesthésiologistes, de néonatologistes, de généticiens et d'échographistes. Le bloc opératoire devient encombré avec deux équipes chirurgicales complètes et deux lots d'instruments chirurgicaux. Un équipement adéquat doit être facilement disponible. Cet équipement comprend une agrafeuse utérine spécialement conçue avec des agrafes résorbables ${ }^{12}$ afin d'empêcher l'hémorragie pendant la période prolongée d'atonie utérine.

Chaque membre de l'équipe joue un rôle vital. L'obstétricien doit éviter d'endommager le placenta et prévoit l'incision utérine après un repérage minutieux par échographie de la position des marges du placenta. Après l'hystérotomie, la visibilité du foetus rend possible une anesthésie foetale im supplémentaire, si nécessaire. Une fois la tête du foetus exposée, l'accès aux voies respiratoires est assuré par une bronchoscopie rigide après exposition du larynx. Selon l'indication de la procédure EXIT, le foetus peut être exposé comme nécessaire. L'échographie peut être utilisée pendant l'opération pour surveiller les pulsations du cordon ombilical et la fonction cardiaque fotale. Après l'opération, le cordon ombilical est coupé et le nouveau-né est confié au néonatologiste pour un examen et un traitement approfondis.

En outre, des questions éthiques peuvent survenir. Le risque pour la mère doit être soupesé par rapport aux avantages potentiels pour le nouveau-né, en particulier s'il présente d'autres anomalies ou déficiences génétiques graves. Dans chaque cas, une discussion impliquant tous les membres de l'équipe susmentionnée doit avoir lieu. Un diagnostic morphologique et génétique est important afin d'assurer que l'intervention n'est pas effectuée sur un foetus souffrant d'autres anomalies létales.

La procédure EXIT est relativement récente, et est prometteuse pour les nouveau-nés souffrant d'obstruction des voies respiratoires. L'anesthésiant peut être administré de différentes manières, pourvu que l'oxygénation foetale et la relaxation utérine soient maintenues. La planification préopératoire et la communication sont les facteurs essentiels pour déterminer les ressources nécessaires avec succès. Les questions éthiques doivent être prises en considération afin d'optimiser les bienfaits pour la mère et le fotus. 


\section{References}

1 George RB, Melnick AH, Rose EC, Habib AS. Case series: combined spinal epidural anesthesia for cesarean delivery and ex utero intrapartum treatment (EXIT) procedure. Can J Anesth 2007; 54: 218-22.

2 Norris MC, Joseph J, Leighton BL. Anesthesia for perinatal surgery. Am J Perinatol 1989; 6: 39-40.

3 Marwan A, Crombleholme TM. The EXIT procedure: principles, pitfalls, and progress. Sem Pediatr Surg 2006; 15: 107-15.

4 Rosen MA. Anesthesia for fetal procedures and surgery. Yonsei Med J 2001; 42: 669-80.

5 Restrepo CE, Gomez ME, Puerta JJ, Upegui A. Anaesthesia for the EXIT procedure: the value of fastrack anaesthesia. Int J Obstet Anesth 2006; 15: S43.

6 Ngan Kee WD, Khaw KS. Vasopressors in obstetrics: what should we be using? Curr Opin Anaesthesiol 2006; 19: 238-43.

7 Haydon ML, Gorenberg DM, Nageotte MP, et al. The effect of maternal oxygen administration on fetal pulse oximetry during labor in fetuses with nonreassuring fetal heart rate patterns. Am J Obstet Gynecol 2006; 195: 735-8.

8 Hirose $S$, Harrison MR. The ex utero intrapartum treatment (EXIT) procedure. Semin Neonatol 2003; 8: 207-14.

9 Lee SJ, Ralston HJ, Drey EA, Partridge JC, Rosen MA. Fetal pain: a systematic multidisciplinary review of the evidence. JAMA 2005; 294: 947-54.

10 Bouchard S, Johnson MP, Flake AW, et al. The EXIT procedure: experience and outcome in 31 cases. $\mathrm{J}$ Pediatr Surg 2002; 37: 418-26.

11 Scully Noah MM, Norton ME, Sandberg P, Esakoff T, Farrell J, Albanese CT. Short-term maternal outcomes that are associated with the EXIT procedure, as compared with cesarean delivery. Am J Obstet Gynecol 2002; 186: 773-7.

12 Hirose S, Farmer DL, Lee H, Nobuhara KK, Harrison $M R$. The ex utero intrapartum treatment procedure: Looking back at the EXIT. Journal of Pediatric Surgery 2004; 39: 375-80. 